\title{
SPECTRAL ENERGY DISTRIBUTIONS OF $Z>2$ GALAXIES IN THE HUBBLE DEEP FIELD
}

\author{
MARCIN SAWICKI \& H.K.C. YEE \\ Department of Astronomy, University of Toronto \\ 60 St.George Street, Toronto, Ontario M5S 3H8, Canada
}

Broadband spectral energy distributions $\left(V_{606}, I_{814}, J, H, K_{s}\right)$ of 17 spectroscopically confirmed $z>2$ Hubble Deep Field galaxies were compared with spectral synthesis models wich had been corrected for dust using the reddening law for star-forming regions. Sawicki \& Yee (AJ submitted) contains the detailed description, while here we summarise the main results.

We find that the spectroscopically confirmed Lyman break galaxies in the Hubble Deep Field:

1. Are dominated by very young (typically $<0.2 \mathrm{Gyr}$ ) stellar populations (see Figure below). The absence of older populations implies that star formation at high $z$ is episodic rather than continuous.

2. Have enough dust to suppress rest-frame UV flux by a factor of $>10$.

3. Have large star formation rates - typically $60 h^{-2} M_{\odot} y r^{-1}$ (see Fig.).

4. Produce, in a typical star-forming episode, $\frac{1}{15}-\frac{1}{20}$ the stellar mass contained in a present-day $L^{*}$ galaxy (see Fig.).
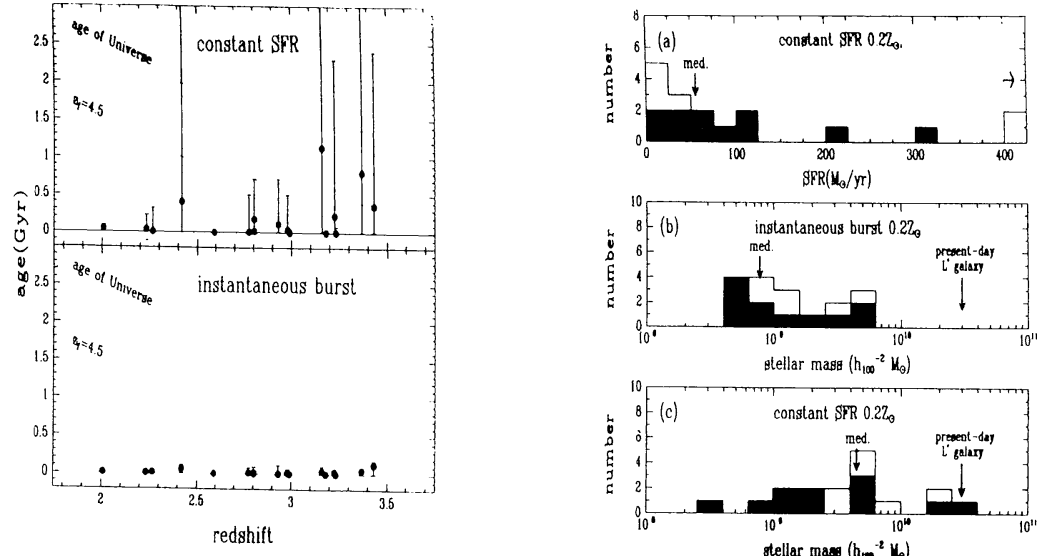

stellar mass $\left(\mathrm{h}_{100}{ }^{-2} \mathrm{~h}_{\theta}\right)$

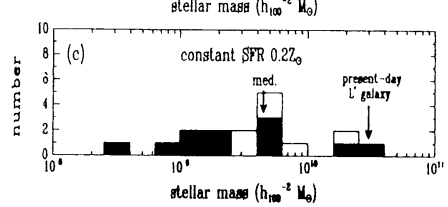

Figure 1. Left: Ages of dominant stellar populations from constant SFR and instantaeous burst fits. Right: Star formation rates and resultant masses of stellar populations. 